\title{
Usefulness of Chest Computed Tomography for Diagnosis of Idiopathic Pneumomediastinum with Negative Findings on Plain X-Ray ${ }^{*}$
}

\author{
Kazuhiro Mino" ${ }^{1}$, Tadao Okada ${ }^{2 \#}$, Shohei Honda ${ }^{2}$, Hisayuki Miyagi ${ }^{2}$, Akinobu Taketomi ${ }^{1}$ \\ ${ }^{1}$ Department of Gastroenterological Surgery I, Graduate School of Medicine, Hokkaido University, Sapporo, Japan \\ ${ }^{2}$ Department of Pediatric Surgery, Hokkaido University Hospital, Sapporo, Japan \\ Email: \#okadata@med.hokudai.ac.jp
}

Received December 6, 2011; revised December 30, 2011; accepted January 10, 2012

\begin{abstract}
Idiopathic pneumomediastinum is rare in children. Few cases of patients with pneumomediastinum show negative findings on X-ray examination. Chest computed tomography (CT) was very useful for the diagnosis and evaluation of the extent of pneumomediastinum. We report here a case of idiopathic pneumomediastinum in a 15-year-old boy who exhibited no significant chest X-ray finding. The patient was referred to our institute for the further evaluation of precordial pain and breathing difficulty. Precordial pain suddenly developed, when he was carrying a portable shrine on his shoulder (day of onset). He was admitted to another institute 3 days after onset because of worsening precordial pain. On admission, he presented with $98 \%$ saturation of hemoglobin in the peripheral blood under room air. Plain chest X-ray also revealed no abnormal findings. A half-dissolved gastrographin swallow showed no leakage of gastrographin from the pharynx and esophagus to the mediastinum, and no diverticulum within the esophagus. Plain chest CT revealed extensive emphysema around the trachea from the neck to the portion inferior to the carina of trachea. The patient was diagnosed with idiopathic pneumomediastinum because the cause was unclear. We decided to admit him to our institute under fasting conditions and rest. His symptoms improved 3 days after onset. The lesion had disappeared 8 days after onset on chest CT. When young people experience precordial pain which increases on inspiration, we must consider pneumomediastinum in a differential diagnosis, and it is important to perform chest CT.
\end{abstract}

Keywords: Chest X-Ray; Child; Computed Tomography (CT); Idiopathic Pneumomediastinum

\section{Introduction}

Pneumomediastinum generally occurs in association with trauma, surgical stress, or basal lung disease, and idiopathic pneumomediastinum, which is not caused by underlying disease and has no known cause, is rare in children. In addition, most patients with pneumomediastinum, associated X-ray findings, such as a vertical lucent streak along the left side of the heart, are positive [1,2]. However, a few pneumomediastinum patients show negative findings on X-ray examination. We report here a case of idiopathic pneumomediastinum in a 15-year-old boy showing no significant chest X-ray finding, whereby chest computed tomography (CT) was very useful for the diagnosis and evaluation of the extent of idiopathic pneumomediastinum. We review the literature on this type of disorder and discuss the problems associated with the diagnosis of children with idiopathic pneumomediastinum, particularly concerning the usefulness of chest CT.

\footnotetext{
"Source of Support: Nill; Conflict of Interest: None declared.

"Corresponding author.
}

\section{Case Report}

A 15-year-old boy was referred to our institute for the further evaluation of precordial pain and breathing difficulty. There was no history of chest pain or dyspnea. Precordial pain suddenly developed, when he was carrying a portable shrine on his shoulder (day of onset). He was admitted to another institute 3 days after onset because of worsening precordial pain and suspected pneumomediastinum. He was referred to our institute the same day for further evaluation and treatment. On admission, his height was $176.3 \mathrm{~cm}$ and weight $69.6 \mathrm{~kg}$ (body mass index; 22.5). The patient's vital signs were: body temperature 36.5 degrees, blood pressure 110/55 $\mathrm{mmHg}$, pulse rate $70 / \mathrm{min}$, and respiratory rate $15 / \mathrm{min}$. He presented with $98 \%$ saturation of hemoglobin in the peripheral blood under room air. On auscultation, abnormal sounds were not noted in his lungs and heart. Precordial pain increased on taking a deep breath. His blood chemistry values were within normal limits: white blood cell count $5600 / \mathrm{mm}^{3}$; C reactive protein $<0.24$ 
mg/dl). Electrocardiogram showed no abnormal findings. Plain chest X-ray also revealed no abnormal findings (Figure 1). A half-dissolved gastrographin swallow showed no leakage of gastrographin from the pharynx and esophagus to the mediastinum, and no diverticulum within
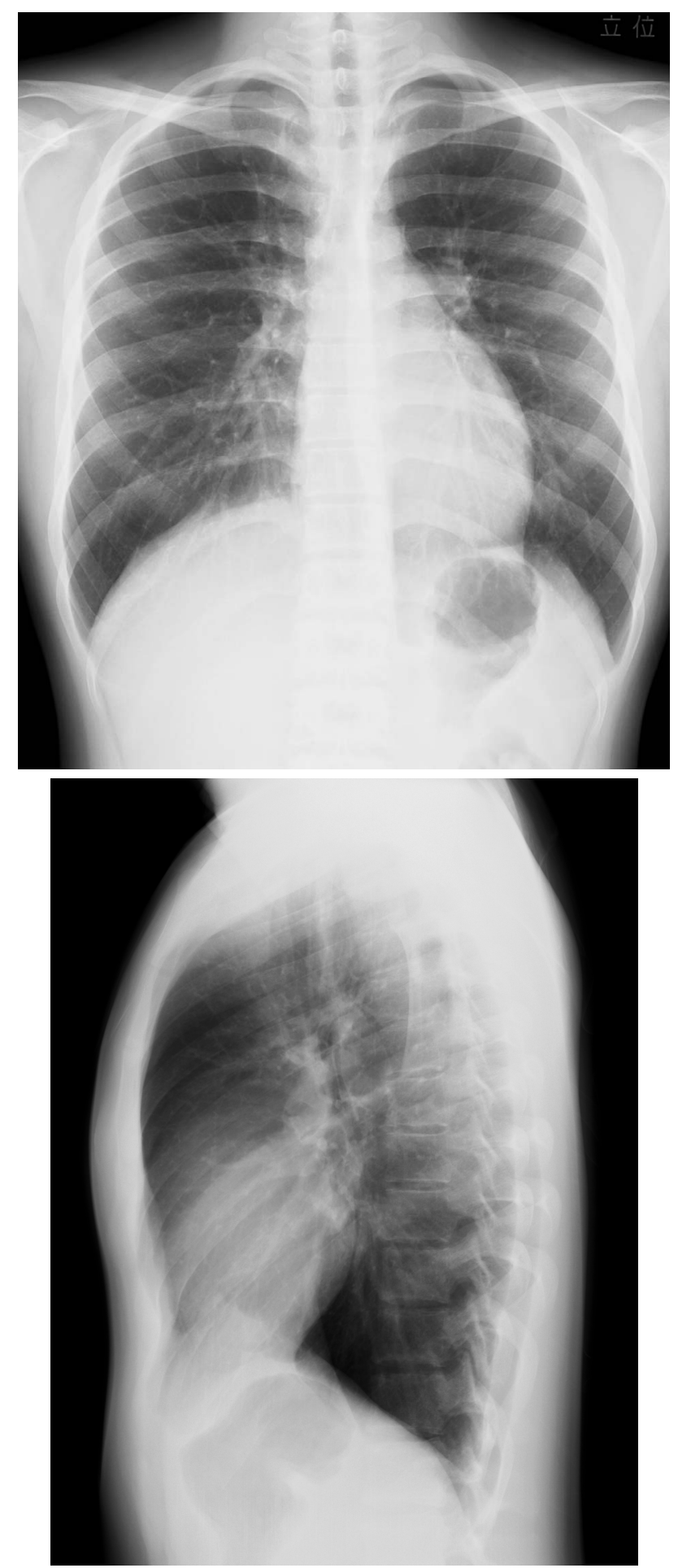

Figure 1. Anterior-posterior and lateral chest $\mathrm{X}$-ray films on admission show no emphysema in the mediastinum. the esophagus (Figure 2). Plain chest CT revealed extensive emphysema around the trachea from the neck to the portion inferior to the carina of trachea (Figure 3). The patient was diagnosed with idiopathic pneumomediastinum because the cause was unclear. We decided to admit him to our institute under fasting conditions and rest. His symptoms improved 3 days after onset. Limited oral feeding was started 4 days after onset, and full oral feeding began 5 days after onset. The lesion had disappeared 8 days after onset on chest CT (Figure 4). He was discharged asymptomatically 9 days after onset. He has had no recurrence of pneumomediastinum for 6 years.

\section{Discussion}

Pneumomediastinum generally occurs in association with

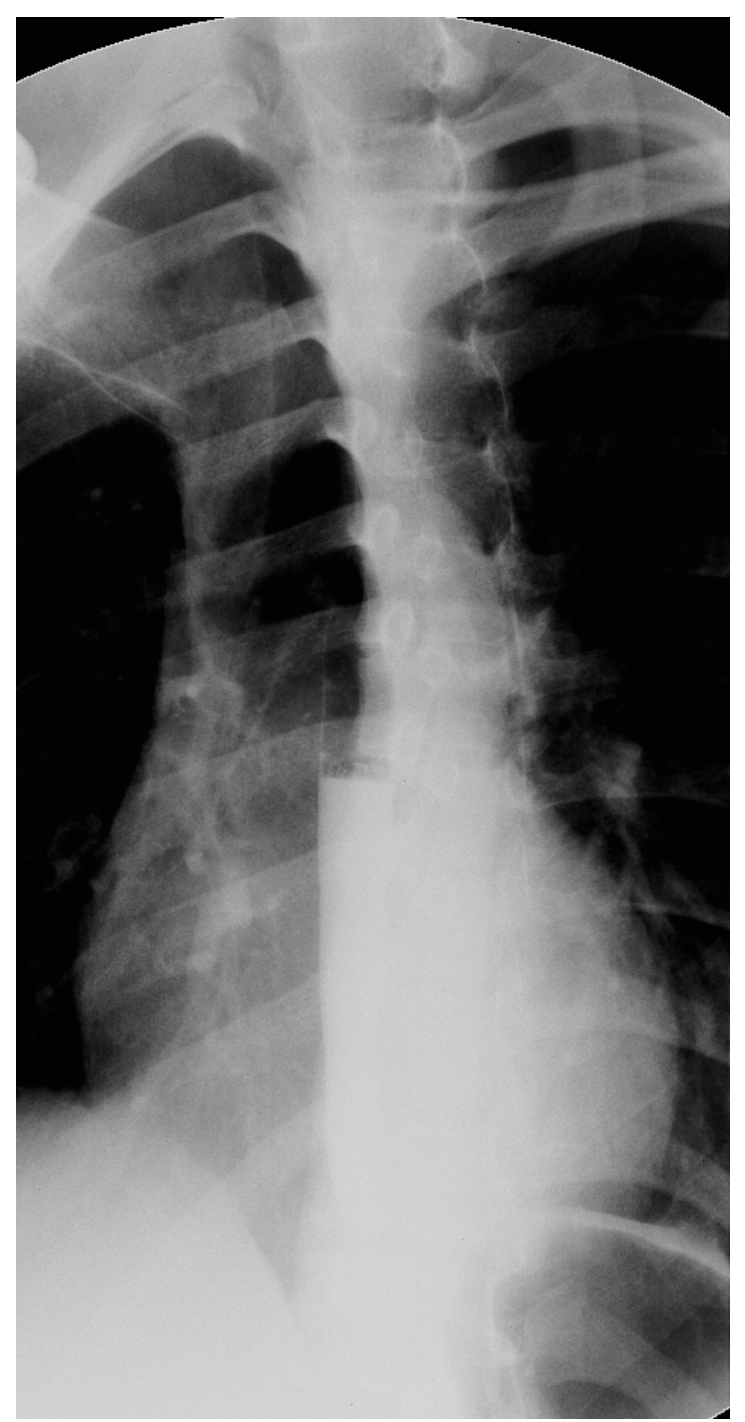

Figure 2. Half-dissolved gastrographin swallow study shows no evidence of leakage of gastrographin from the pharynx and esophagus to the mediastinum, and no diverticulum within the esophagus are observed. 

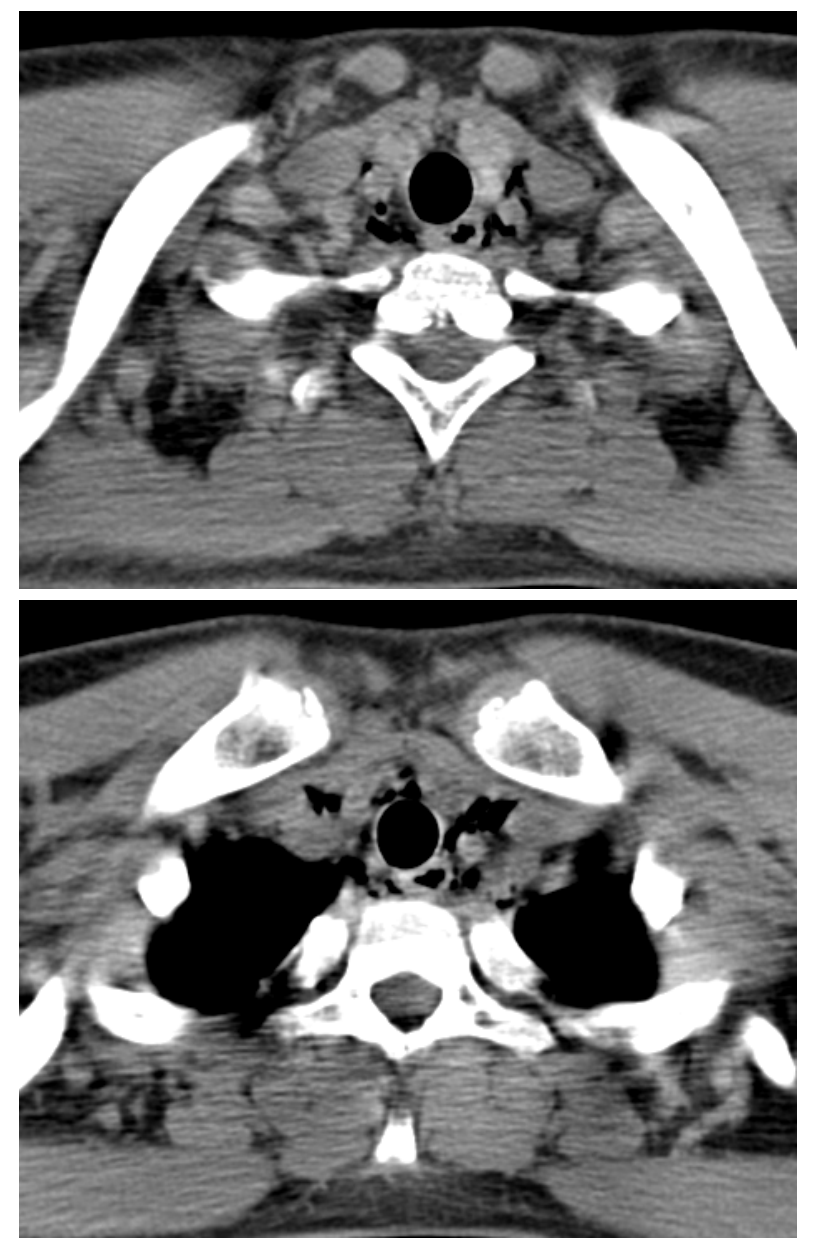

Figure 3. Chest CT on admission shows emphysema (arrows) from the superior mediastinum to the portion inferior to the carina. As the result, pneumomediastinum is shown on the CT although chest X-ray films on admission show no emphysema in the mediastinum.

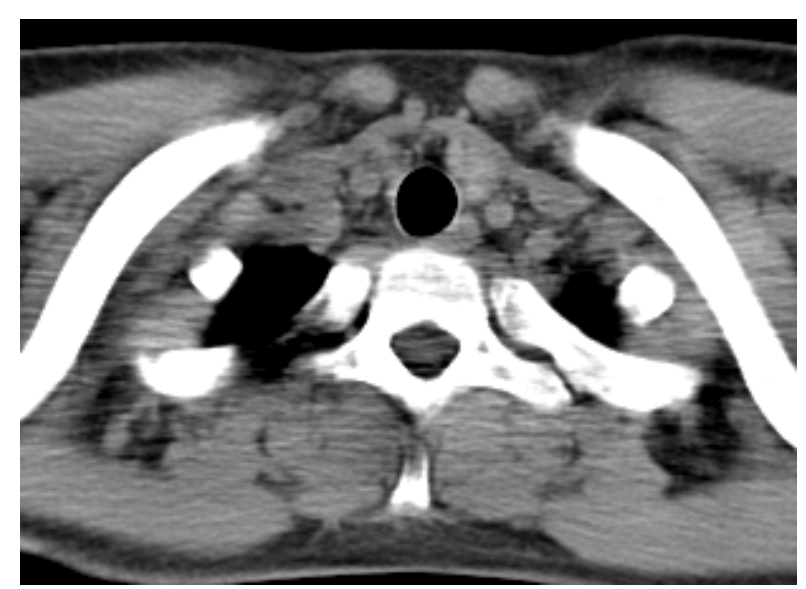

Figure 4. The lesion of pneumomediastinum disappeared 8 days after onset on chest $\mathrm{CT}$.

trauma, surgical stress, or basal lung disease. Idiopathic pneumomediastinum for the first time in 1939, and has no clear cause or basal disease. However, so-called idiopathic pneumomediastinum generally includes pneumomediastinum with increased intrapleural pressure caused by exercise and cough. According to an actual trigger cause, it is described that $30 \%$ of the cases had no trigger, $62 \%$ occurred during sports, and $8 \%$ with vocalization. In our case, it was suggested that intra-alveolar pressure increased while carrying the portable shrine on his shoulder, possibly inducing alveolar wall burst. Therefore, it is thought that our case was similar to those occurring during sports. Spontaneous pneumomediastinum (SPM) and idiopathic pneumomediastinum are often confused. Many reports on idiopathic pneumomediastinum include SPM and many reports on SPM include cases caused by bronchial asthma and upper respiratory infection. Joyaube et al described $49 \%$ cases of SPM caused by bronchospasm, $21 \%$ were from respiratory infection, $8 \%$ followed foreign body inhalation, and the frequency of "real" idiopathic pneumomediastinum, such as in our case, comprised only $15 \%$ of SPM cases [1]. Therefore, real idiopathic pneumomediastinum is extremely rare.

Abolnik et al. reported that it had a prevalence of one patient per 1800 - 42,000 emergency visits [3,4], while a report from a United States Armed Forces hospital, which many young men visit, stated that it occurred in one patient per 800 - 7115 visits [5,6].

On physical examination, atypical symptoms such as difficulty of breathing, coughing, neck pain, and subcutaneous emphysema are present in about $60 \%-80 \%$ of cases. But, Hamman's symptom, a famous and typical mediastinal friction murmur in accordance with cardiac beat, which can be heard from the left sternal border to the apex of the heart, is present in $20 \%$ - $40 \%$ of cases [2]. According to Tanaka et al., this murmur is heard when the emphysema reaches the chest wall on the anterior aspect of the cardiac ventricle. Based on these findings, physical findings are not enough to facilitate the diagnosis of pneumomediastinum.

The standard modality for the diagnosis of pneumomediastinum is plain chest frontal and lateral X-ray, and by this modality, $91 \%$ of 33 patients with pneumomediastinum, $98 \%$ of 53 , and $100 \%$ of 58 could be diagnosed [1]. However, there are patients whose findings on plain chest $\mathrm{X}$-ray are not significant, such as the present case. Therefore, chest CT is required when there is precordial pain which increases on inspiration. We doubted the present of SPM based on the clinical symptoms in the present case and performed chest CT, which revealed peritracheal air density and led to a diagnosis. In this way, even if the chest X-ray findings are negative, CT is useful for the diagnosis of pneumomediastinum. For the evaluation of the extent of pneumomediastinum, there is a report that free air in the mediastinum is initially located in the upper lesion, and then invades the middle and lower le- 
sion with the progression of the disease, and the state of the disease can be classified on chest CT: Grade A = free air confined to the superior portion of the mediastinum only; grade $\mathrm{B}$ = free air extending from the superior portion of the mediastinum to the middle portion of the mediastinum; grade $\mathrm{C}=$ free air extending from the superior to the inferior portion of the mediastinum. The superior portion of the mediastinum was defined as the portion superior to the carina, the middle portion of the mediastinum as that from the level of the carina to the orifice of the inferior pulmonary vein, and the inferior portion of the mediastinum as that inferior to the pulmonary vein. On the basis of this classification, the present case is grade $\mathrm{B}$. We should be careful to recognize grade $\mathrm{B}$ cases, even if the chest $\mathrm{X}$-ray findings are negative. On the findings of electrocardiography for pneumomediastinum, atypical changes are found in only about $25 \%$ of cases [7], and the diagnostic value of electrocardiography is not clear.

Treatment is generally only bed rest, often with transient symptom exacerbation [3], and almost all patients are cured within two weeks. If infectious signs are not clear, antibiotics are not needed. We did not use antibiotics in our case too, and the patient could be discharged from the hospital on the 9th day after onset.

Concerning the recurrence rate, a study reported a rate of $4.3 \%$ in SPM [2], but in many other reports, the recurrence rate is extremely low. The present case is currently well without recurrence.

\section{Conclusion}

In conclusion, a few pneumomediastinum patients have been reported to show negative X-ray findings, and we could diagnose it by additionally performing chest CT.
When young people complain of precordial pain which increases on inspiration, we must consider SPM in a differential diagnosis. Chest CT is useful for the diagnosis and evaluation of the extent of pneumomediastinum, especially in children with negative findings on plain chest X-ray.

\section{REFERENCES}

[1] C. Joyaube, B. Mona, D. Patrick, B. Denis, B. Andreana, O. Alain, et al., "Spontaneous Pneumomediastinum: Are We Over-Investigating?” Journal of Pediatric Surgery, Vol. 39, No. 5, 2004, pp. 681-684. doi:10.1016/j.jpedsurg.2004.01.045

[2] I. Abolnik, I. S. Lossos and R. Breuer, "Spontaneous Pneumomediastinum: A Report of 25 Cases,” Chest, Vol. 100, No. 1, 1991, pp. 93-95. doi:10.1378/chest.100.1.93

[3] M. Chalumeau, L. L. Clainche, N. Sayeg, N. Sannier, J. L. Michel, R. Marianowski, et al., "Spontaneous Pneumomediastinum in Children,” Pediatricic Pulmonology, Vol. 31, No. 1, 2001, pp. 67-75. doi:10.1002/1099-0496(200101)31:1<67::AID-PPUL100 9>3.0.CO;2-J

[4] A. Yellin, M. Gapany and Y. Lieberman, "Spontaneous Pneumomediastinum,” Chest, Vol. 101, No. 6, 1992, 1742-1743. doi:10.1378/chest.101.6.1742b

[5] W. P. Munsell, "Pneumomediastinum. A Report of 28 Cases and Review of the Literature," Journal of the American Medical Association, Vol. 202, No. 8, 1967, pp. 689-693. doi:10.1001/jama.1967.03130210063009

[6] D. J. McMahon, "Spontaneous Pneumomediastinum," American Journal of Surgery, Vol. 131, No. 5, 1976, pp. 550-551. doi:10.1016/0002-9610(76)90008-8

[7] M. Aisner and J. E. Franco, "Mediastinal Emphysema," New England Journal of Medicine, Vol. 241, No. 11, 1949, pp. 818-825. doi:10.1056/NEJM194911242412105 